Semin Neurol. 2011 November ; 31(5): 449-460. doi:10.1055/s-0031-1299784.

\title{
Genetics of Dementia
}

\author{
Henry L. Paulson, M.D., Ph.D. ${ }^{1}$ and Indu Igo, Ph.D. ${ }^{1}$ \\ ${ }^{1}$ Department of Neurology, University of Michigan, Ann Arbor, Michigan.
}

\section{Abstract}

Genetic factors are now recognized to play an important role in most age-related dementias. While other factors, including aging itself, contribute to dementia, in this review we focus on the role of specific disease-causing genes and genetic factors in the most common age-related dementias. We review each dementia within the context of a genes/environment continuum, with varying levels of genetic versus environmental influence. All major classes of dementia will be discussed but greatest attention will be given to the most common dementia, Alzheimer's disease, for which several new genetic factors were recently identified.

\section{Keywords}

Amyloid; frontotemporal dementia; dementia with Lewy bodies; neurodegeneration; genetic risk factors

\section{AGE-RELATED DEMENTIAS ARE PROTEINOPATHIES WITH VARIABLE GENETIC INFLUENCE}

It has become increasingly clear that most age-related neurodegenerative diseases, including virtually all dementias, map to various classes of "proteinopathy." The term proteinopathy refers to the tendency for specific proteins to abnormally accumulate and deposit within the brain, often within specific neuropathological structures. Stated differently, all the major types of dementias represent protein conformational disorders. The key classes of dementing proteinopathy are the amyloidopathies, tauopathies, synucleinopathies, TDP43-opathies, prionopathies, and polyglutaminopathies.

Except for the strictly heritable polyglutamine diseases, all of these classes of proteinopathy can be familial (i.e., hereditary), in which case they are caused by dominant-acting disease gene mutations, or sporadic (i.e., nonhereditary) in which case they are caused by a multitude of factors, including advancing age. While not strictly genetic disorders, many sporadic dementias are made more or less likely by the presence or absence of specific genetic risk factors. As illustrated in Figure F, the full spectrum of the genes versus environment continuum (Fig. 1) is covered by the dementias. At one end, the "pure" genetic disorders (e.g., Huntington's disease and other polyglutamine diseases) are still influenced modestly by environmental factors (such as degree of physical activity). At the other end, acquired disorders (e.g., the chronic traumatic encephalopathy recently described in some football players) will likely prove to be influenced by genetic factors. A disease like Alzheimer disease's (AD) falls squarely in the middle of this genes/environment continuum.

Copyright (C) 2011 by Thieme Medical Publishers, Inc.

Address for correspondence and reprint requests: Henry L. Paulson, M.D., Ph.D., Professor of Neurology, Department of Neurology, University of Michigan, 4001 BSRB, 109 Zina Pitcher Place, Ann Arbor, MI 48109 (henryp@ umich.edu).. 
Overlap among the major classes of proteinopathies has also become evident. This is illustrated best by $\mathrm{AD}$, which by definition is both an amyloidopathy and a tauopathy. Likewise, in Lewy body dementia the widespread deposits of a-synuclein in Lewy bodies are frequently accompanied by amyloid plaques. Molecular studies also suggest biological interactions between a-synuclein and amyloid/tau pathways in the central nervous system (CNS). And while TDP-43 inclusions are a hallmark of a large subset of frontotemporal dementia, TDP43 staining also can be seen in select brain regions in other dementias that are not primarily TDP43-opathies. As exacting clinicians, neurologists strive to fit every patient neatly into a diagnostic category, but this is made more challenging by the overlap among the neurodegenerative proteinopathies. Indeed, recent neuropathological series indicate that mixed-type dementia is much more common than was once thought.

\section{Alzheimer's Disease}

Alzheimer's disease is by far the most common form of age-related dementia. Alzheimer's disease is classified as early onset versus late onset, with 65 years being the dividing threshold (for review, see ${ }^{1-3}$ ). Alzheimer's disease is a slowly progressive disease that usually manifests first as amnestic mild cognitive impairment (MCI) before progressing to full-blown dementia, in which memory impairment usually remains the most prominent cognitive deficit. Cerebral atrophy in $\mathrm{AD}$ is accompanied by two key neuropathological hallmarks: amyloid plaques composed of $\beta$-amyloid and neurofibrillary tangles composed of the microtubule-associated protein, tau.

Early onset $\mathrm{AD}$ is often caused by mutations in specific genes that cause autosomal dominant dementia in the family, also known as familial AD. Three different dominantacting disease genes have been implicated. The amyloid precursor protein gene ( $A P P)$, discovered first, encodes the neuronal membrane protein APP from which $\beta$-amyloid (A- $\beta$ ) is released through the sequential action of two proteases, $\beta$-secretase and gamma-secretase. The other two genes underlying familial AD are presenilins 1 and 2. Presenilins are a key component of the gamma-secretase complex. Presenilin 1 mutations are the most common cause of familial AD. Mutations in APP are much less common, and mutations in presenilin 2 rarer still. Collectively, families with familial AD from these three disease genes comprise only $\sim 2 \%$ of all AD. Nevertheless, their discovery has greatly advanced understanding of the pathophysiology of all AD.

The overwhelming majority of $\mathrm{AD}$ is late onset $\mathrm{AD}$. Strictly speaking, late onset $\mathrm{AD}$ is not a genetic disorder, although genetic factors play an important role. The most important genetic risk factor in late onset AD is Apolipoprotein E (ApoE), which consistently surfaces as the biggest genetic risk factor in association studies of $\mathrm{AD}$ (for review, see ${ }^{4}$ ). The ApoE E4 allele is associated with an increased risk of late onset $\mathrm{AD}$ and the ApoE E2 allele with reduced risk. As a genetic risk factor rather than a disease-causing mutation, the ApoE4 allele is neither necessary nor sufficient to cause AD; some people with an ApoE4 allele do not develop AD, and plenty of people without an ApoE4 allele do. Accordingly, at this point the clinical value to an individual in knowing his or her ApoE4 status remains controversial.

While ApoE4 status, together with imaging and cerebrospinal fluid (CSF) biomarkers, can be used to increase the probability of correctly diagnosing $\mathrm{AD}$ or predicting progression from $\mathrm{MCI}$ to $\mathrm{AD}, \mathrm{ApoE}$ allele testing is not currently used routinely by neurologists. A recent study showed, however, that well-informed individuals are generally well equipped to handle this piece of genetic information. ${ }^{5}$

As discussed below, numerous genome-wide association studies have recently uncovered additional genetic risk factors of much lower significance than ApoE. These new factors have not yet entered the clinical realm, but studies are beginning to link specific genetic risk 
factors to one or more components of the AD phenotype (e.g., cognitive profile, structural changes observed on imaging, CSF biomarkers).

While most $\mathrm{AD}$ is not strictly genetic, the three identified disease-causing genes and the ApoE risk factor have led to a much better understanding of key pathogenic events in AD (for review, see ${ }^{6-8}$ ). Together they support the amyloid cascade hypothesis, the prevailing model of AD pathogenesis. For example, APP is the precursor protein for A- $\beta$, and APP mutations typically increase production of A- $\beta$. Some familial AD is due to triplication of this gene, leading to an increased dose of APP. Likewise, persons with Down syndrome, who have a third copy of APP by virtue of trisomy 21, develop age-dependent amyloid deposition and dementia. Both presenilins 1 and 2 are components of gamma-secretase, the protease complex responsible for the final cleavage step that releases A- $\beta$ peptide. Finally, ApoE, the most important genetic factor in late onset AD, is implicated in both the aggregation and clearance of A- $\beta$, with different ApoE isoforms (E2, E3, and E4) having differential activity. ${ }^{4}$

Thus, all three identified disease genes as well as ApoE link to the production or accumulation of amyloid. Implicit in the amyloid cascade model, however, is a biological and temporal connection between early A- $\beta$-mediated events and later tau-mediated events. 9,10 While tau is not itself mutated in $\mathrm{AD}$, mutations in tau do cause another form of dementia, frontotemporal dementia (FTD) or frontotemporal lobar degeneration (FTLD), as described below. Some research findings favor the view that aberrant tau behavior correlates more closely with neuronal death than does amyloid deposition.

The discovery of AD disease genes has spurred the development of biomarkers that can be used to make diagnostic and prognostic predictions. ${ }^{11-18}$ For example, amyloid now can be imaged noninvasively through positron emission tomography (PET) with Pittsburgh compound B (PiB) or similar amyloid imaging compounds. Studies employing PiB have shown that amyloid deposition occurs many years prior to the onset of AD, consistent with the amyloid cascade hypothesis. In general, human studies confirm that production of amyloid is indeed a proximal event in disease. Likewise, a reduction in A- $\beta$ in the CSF correlates with amyloid plaque formation in early stages of disease. Later in disease, an increase in total tau and phosphorylated tau in the CSF correlates with progressive brain atrophy. The eventual successful development of preventive therapies for AD likely may depend on employing such biomarkers in future preventive clinical trials, and currently A- $\beta$ and tau levels in the CSF can be used to increase the accuracy of diagnosing AD versus other forms of dementia.

While modest symptomatic therapies exist for AD, preventive therapies are still lacking. Several recent trials and others currently underway seek to reduce amyloid production/ accumulation or enhance its clearance from the brain. Some AD researchers fear, however, that this approach may be too little too late for those with amnestic MCI or AD. By that point in disease, anti-amyloid therapy may not be sufficient since a key downstream pathological entity, phosphorylated tau, might continue to exert its neurotoxic effects even if accumulated $\beta$ amyloid is eliminated. Eventual therapeutic success may require hitting the amyloid target even earlier in the disease process and/or or attacking both of the key neuropathological targets, amyloid and tau.

Emerging insights from biomarker studies, coupled with the failure thus far to find effective preventive therapies, have led the recent push for scientists and physicians to take a longer view of AD-to view it as a disease process extending over decades rather than narrowly as the dementia which declares itself late in the course of disease progression. ${ }^{19}$ Growing evidence indicates that we can identify biomarkers of ongoing disease well before a person 
shows cognitive symptoms. Testing potential preventive therapies early in the disease process may improve our ability to find effective drugs that slow the rate of disease or stop it altogether.

\section{Susceptibility Genes in Late Onset AD}

Many reports, mostly employing a candidate gene approach, have claimed or refuted hundreds of potential risk genes in $\mathrm{AD} .{ }^{20}$ The non-replication of many of these studies reflects the fact that most were insufficiently powered to detect small genetic effects. ${ }^{21}$ Recently, genome-wide association studies (GWAS), which establish linkage to disease based on SNPs closely spaced across the full genome, have offered a more powerful approach to identify susceptibility genes for complex disorders.

In four recent major GWAS studies of $\mathrm{AD}$, as well as in many earlier association studies, $A P O E$ was identified as a powerful genetic risk factor ${ }^{22-25}$ (for review, see ${ }^{26}$ ). The recent GWAS studies also identified 9 novel loci for which the evidence is compelling: $C L U$, PICALM, CR1, BIN1, ABCA7, MS4A cluster, CD2AP, CD33 and EPHA1. In comparison to $A P O E$, these identified factors confer a relatively small risk for disease: an increased disease odds ratio of only 1.1-1.15. In contrast, the odds ratio for the APOE E4 allele is 3.0 to 4.0 .

The immediate implication of these newly identified susceptibility genes is that they suggest novel mechanisms contributing to disease pathogenesis that may not be linked to the production, oligomerization or clearance of $\beta$-amyloid. As discussed below, these pathways include the immune system and complement activation, synaptic dysfunction, membrane recycling, and lipid metabolism. In the near future, full genomic or exomic sequencing of affected versus control cases likely will contribute still further to our understanding of genetic contributions to late onset AD.

The newly identified genetic risk factors are:

\section{CLU}

$C L U$ was identified as a risk factor in multiple GWAS analyses. ${ }^{22,23}$ The $C L U$ gene product, clusterin, is an apolipoprotein expressed in most tissues including the brain. Several properties of clusterin suggest a link to A- $\beta$ : 1 ) it is present in amyloid plaques; 2 ) it acts as an $A-\beta$ chaperone to block aggregation; 3 ) it mediates A- $\beta$ clearance at the blood brain barrier by increasing glial endocytosis; and 4 ) it regulates amyloid formation and cytotoxicity. ${ }^{27}$ Clusterin also plays a role in inflammation and the immune response, inhibiting complement activation ${ }^{28}$ and modulating the inflammatory response associated with complement activation downstream of A- $\beta$ aggregation. In addition, elevated plasma levels of clusterin have been observed in $\mathrm{AD},{ }^{29}$ its expression is increased in affected cortical areas, and it is found in the CSF of AD patients. ${ }^{30}$ The disease-associated $C L U \mathrm{C}$ allele is associated with impaired white matter integrity. ${ }^{31}$

\section{CR1}

$\mathrm{CR} 1$ is linked to $\mathrm{AD}$ through $\mathrm{A}-\beta$-induced activation of the $\mathrm{C} 3$ complement cascade. The $C R 1$ gene resides on chromosome 1 amid the gene cluster of regulators of complement activation. ${ }^{32} \mathrm{~A}$ crucial mediator of innate immunity, CR1 functions as an immune adherence molecule on erythrocytes and is abundant in neurons of the AD brain. ${ }^{33}$ For years, neuroinflammation and activation of the complement system have been suspected to play a role in $\mathrm{AD}$ pathology. ${ }^{34} \mathrm{CR} 1$ could modulate $\mathrm{AD}$ pathogenesis at several levels. It could alter the clearance of A- $\beta$ since circulating A- $\beta$ is cleared by $C 3 b$-mediated adherence to 
CR1 at the erythrocyte surface, a process which is decreased in AD patients. ${ }^{35}$ It also could participate in disease as a negative regulator of the complement cascade, immune adherence, and/or phagocytosis. Genetic association of CR1 with AD risk may be explained by copy number variation (CNV) that results in two major isoforms differing in the number of $\mathrm{C} 3$ / $\mathrm{C} 4 \mathrm{~b}$ and cofactor activity sites, and thus having different functional roles in the complement cascade. $^{36}$

\section{PICALM}

PICALM encodes a phosphatidylinositol-binding clathrin assembly protein that is expressed at neuronal synapses. ${ }^{37}$ PICALM and its homologue, AP180, likely participate in clathrin assembly on coated pits. Both genes participate in axonal development and dendritic growth, and can alter the size of clathrin-coated vesicles and endosomes. The precise role of PICALM in AD pathophysiology is unclear. It may modulate APP processing through its activity in clathrin-mediated endocytosis (CME). Full length APP is retrieved from the cell surface by CME, and inhibition of endocytosis reduces APP internalization and A- $\beta$ production. ${ }^{38,39}$ PICALM also participates in intracellular trafficking of vesicle associated membrane protein2 (VAMP2) which regulates synaptic vesicle fusion and memory formation. ${ }^{40}$ It is conceivable that the AD-associated PICALM allele promotes synaptic dysfunction through an effect on synaptic cycling, resulting in neurodegenerative changes.

\section{BIN1}

\section{ABCA7}

Bridging Integrator 1 is a tumor suppressor that has long been known to control transit through the cell cycle. ${ }^{41}$ Also known as Amphiphysin 2, BIN1 occurs as several alternatively spliced isoforms, including a brain-specific isoform. Some BIN1 isoforms participate in dynamin-mediated synaptic endocytosis, ${ }^{42}$ and mice lacking BIN1 expression have defective endocytic protein scaffolds and synaptic recycling. Thus, these results implicate BIN1 in receptor mediated endocytosis (RME) and vesicular trafficking as well as the cell cycle. Amyloid precursor protein, Amyloid $\beta$, and APOE are all internalized through endolysosomal trafficking, suggesting a route by which BIN1 functions as a risk factor in AD. Alternatively, because BIN appears to link the microtubule cytoskeleton to the cellular membrane via tubular membrane structures, ${ }^{43}$ it may influence the formation of neurofibrillary tangles, the second major hallmark of AD pathology.

The ATP-binding cassette transporter (ABC) $\mathrm{A} 7$ is an $\mathrm{ABC}$ family protein that transports a wide range of substances across the cell membrane. It is highly homologous to ABCA1, which mediates the biogenesis of high-density lipoprotein (HDL), and is associated with phagocytosis regulated by sterol regulatory element binding protein 2 . ABCA7 may be a key molecule linking sterol homeostasis and the host defense system. ${ }^{44}$ In the human brain, microglia express the highest level of ABCA7 mRNA, 10 fold higher than in neurons. ${ }^{45,46}$ ABCA7 is an ortholog of ced-7 in Caenorhabditis elegans, which is known to clear apoptotic cells. As microglia actively phagocytose apoptotic debris in the CNS, high levels of ABCA7 in the microglia point to a potential role for ABCA7 in phagocytosing apoptotic debris during development or neurodegenerative diseases like $\mathrm{AD}$. ABCA7 has also been shown to regulate APP processing and inhibit A- $\beta$ secretion in cells overexpressing APP. ${ }^{47}$

\section{MS4A}

The MS4A genes are clustered on chromosome 11 in humans. Expressed in lymphoid tissue and brain, they comprise a gene superfamily named the Membrane Spanning 4 domains subfamily A. ${ }^{48}$ In the MS4A gene cluster, multiple variants are associated with 
susceptibility to AD: MS4A2, MS4A3, MS4A4, MS4A4E, MS4A6A and MS4A6E. ${ }^{25}$ A role in innate and adaptive immunity has been shown for several members of this cluster, including MS4A1, MS4A2 and MS4AB. For example, MS4A2 encodes the $\beta$ subunit of the high affinity immunoglobulin epsilon (IgE) receptor. It initiates the allergic response to allergen, leading to cell activation and release of mediators. A link between MS4A2 and AD would further support a pathogenic role for neuro-inflammation in the pathology of AD.

CD33

CD33 is a member of the sialic acid binding immunoglobulin like lectins, also known as SIGLEC3. It encodes a cell surface receptor on cells of myeloid lineage that is thought to promote cell-cell interactions and mediate endocytosis through a clathrin-independent mechanism. ${ }^{49}$ It regulates functions of cells in the innate and adaptive immune systems. ${ }^{50}$

\section{CD2AP}

$\mathrm{CD} 2$ associated protein is a member of a family of related adaptor proteins ${ }^{51}$ with many predicted functions. Among its properties, CD2AP associates with cortactin, a protein involved in the regulation of receptor mediated endocytosis. ${ }^{52}$ As a cortactin binding partner, CD2AP functions similarly to the related $C$. elegans protein CIN85, suggesting a novel link between the endocytic machinery and the actin cytoskeleton that may regulate the trafficking of receptor-containing vesicles in neurons. The CD2AP-cortactin complex possibly regulates the internalization and/or intracellular trafficking of a variety of transmembrane proteins. ${ }^{52}$

\section{NEW PATHOPHYSIOLOGICAL PATHWAYS IMPLICATED BY LATE ONSET AD GENES}

While not rejecting or refuting the amyloid cascade hypothesis, the recent GWAS studies implicate additional regulatory pathways contributing to the etiology of late onset $\mathrm{AD}$ (for additional discussion of non-amyloid pathways implicated in $\mathrm{AD}$, see ${ }^{53}$ ).

\section{Immune System and Neuroinflammation (CLU, CR1, CD33, ABCA7 and MS4A)}

Signs of chronic inflammatory reaction are seen in areas affected by $\mathrm{AD}$, and upregulation of the components of the complement system are observed in neurofibrillary tangles and neuritic plaques. While these phenomena may represent a local response to neuronal damage and aggregated proteins, evidence suggests that they are not merely a byproduct of a pathological cascade. Inflammatory processes potentially could turn a relatively slowly progressive condition into rapid neurodegeneration. ${ }^{54,55}$ Several of the late onset AD genes (ApoE, CLU, CR1, CD33, ABCA7 and MS4A) are involved directly or indirectly in the regulation of inflammatory mechanisms. This further supports a prominent role for the immune system and neuroinflammation in AD pathogenesis.

CLU and CR1 are both implicated in the immune system and may modulate important inflammatory processes in AD. They operate in $\mathrm{A} \beta$ clearance through phagocytosis by immune cells. ${ }^{56}$ Astrocytes are the main source of secreted CLU in brain and ApoE is its main binding partner. Like ApoE, CLU is involved in the clearance of $A \beta$ from the brain through lipoprotein receptors. Clusterin binding may represent a way to protect cells against aggregating proteins by binding to fibrillar proteins. Evidence for clusterin clearing $A \beta$ into glial cells is seen in mouse models of AD. It inhibits complement activation ${ }^{28}$ and prevents the inflammatory response associated with complement activation downstream of protein aggregation. CLU has been linked to chronic inflammation of the brain, as both $\mathrm{C} 3$ and 
clusterin are found in the same neuritic plaques. ${ }^{33}$ It defends neurons against cytolysis by inhibiting the complement system and modulating membrane attack complex. ${ }^{28}$

Genetic variants in CR1 have been implicated in autoimmune and inflammatory diseases. ${ }^{57}$ Depending upon the number of homologous repeats, CR1 contains multiple extracellular binding sites for $\mathrm{C} 3 \mathrm{~b}$ and $\mathrm{C} 4 \mathrm{~b}$. A- $\beta$ oligomers bound to $\mathrm{C} 3 \mathrm{~b}$ adhere to the erythrocyte and are subsequently cleared from the circulation. Transgenic mouse models overexpressing APP have increased $A \beta$ accumulation and inhibition of $C 3 \mathrm{~b}$, suggesting a protective role of $\mathrm{CR} 1$ in AD. Genetic variation in CR1 might affect AD pathogenesis through neuroinflammation related to local complement inhibition.

Though less well characterized than CLU and CR1, the susceptibility genes CD33, ABCA7, and the MS4A gene family have also been implicated in immune and inflammatory pathways in late onset $\mathrm{AD}$, as mentioned above in the individual descriptions of each genetic factor.

\section{Lipid Metabolism/Cholesterol Dysregulation (ApoE, CLU, BIN1, PICALM and ABCA7)}

Regulation of cholesterol homeostasis is crucial for neurological function. Conversely, dysregulation of cholesterol homeostasis can contribute to neurodegeneration. ${ }^{58,59}$ The susceptibility genes ApoE and clusterin are involved in the formation and transport of lipoprotein particles across the blood brain barrier and share many common properties. Clusterin is a chaperone molecule that binds $A \beta$, lipids, and complement factors. It functions in the transport of cholesterol and phospholipids. ${ }^{30}$ Genetic variations in CLU are associated with altered serum lipid levels and increased intima media thickness of the carotid arteries, ${ }^{60}$ and CLU levels are upregulated in atherosclerosis. ${ }^{61}$ Thus, CLU may modify AD pathogenesis by increasing the risk of cerebrovascular disease and as such accelerate the neurodegeneration process. BIN1 and PICALM may also have a role in the transport of lipids through RME, and their relationship to lipid-mediated events may have a potential role in $\mathrm{AD}$. $\mathrm{ABC}$ transporters are expressed in brain and play a key role in brain lipid transport homeostasis. The expression of the ABCA7 gene, which is regulated by sterol regulatory binding protein-2, promotes the efflux of cholesterol and phospholipids. ABCA7 is one of the closest homologue to ABCA1 and is one of the key molecules linking sterol homeostasis and the host defense system. ${ }^{62}$ Dysregulated trafficking of cholesterol and sterol related compounds promotes the formation of arterial plaques and increases the risk of heart disease and $\mathrm{AD} .{ }^{63}$ Genetic mutations that alter the transporter function could impact lipid transport, brain function, and susceptibility to neurodegeneration.

\section{Synaptic Dysfunction and Endocytosis (PICALM, BIN1, CS33, CLU, CD2AP)}

In $\mathrm{AD}$ the disease process itself probably reflects, in part, an aberration of normal physiological processes at or near the synapse. Amyloid precursor protein is normally proteolytically processed during synaptic activity. It's amino-terminal, soluble APP (sAPP) fragment has neurotrophic properties, and the intracellular domain released by gammasecretase is thought to enter the nucleus and regulate gene expression events. While A- $\beta$ was thought by many simply to be a "waste byproduct" of these sequential proteolytic events, recent studies suggest that A- $\beta$ is normally produced during synaptic activity ${ }^{64}$ and may function to provide a negative feedback signal on excess synaptic activity. In other words, A- $\beta$ production may normally help to maintain synaptic homeostasis. As such, the inevitable day-to-day production of A- $\beta$ at highly active synapses can lead to its accumulation and eventual oligomerization. Maybe this is why the basal brain activity pattern known as the "default pathway" aligns closely with the initial sites of amyloid deposition in AD. While the critical toxic species formed by A- $\beta$ remains uncertain, recent studies suggest that dimers or slightly larger oligomers are the key toxic species. ${ }^{65,66}$ Since oligomerization is a 
concentration-dependent process, excess A- $\beta$ at or just outside the neuronal membrane will favor oligomerization and eventual plaque formation.

Very likely, genetic variants in at least a few of the five genes listed above will alter A- $\beta$ production or clearance via changes in synapse-related events. For example, PICALM localizes to synapses, is likely involved in APP processing through the endocytic pathway, and has a prominent role in trafficking of VAMP2, a protein involved in synaptic vesicle fusion and neurotransmitter release. BIN1 is one of two related amphiphysins that play a critical role in neuronal membrane organization and clathrin-mediated synaptic vesicle formation. BIN1 knockout mice have deficient protein scaffolds and synaptic vesicle recycling, and display seizures and major learning deficits. The involvement of BIN1 in vesicular trafficking could alter dynamics of APP and A- $\beta$ at or near the synapse. CLU binds $A-\beta$ and is involved in the clearance of $A-\beta$ from the brain either by enhancing receptor mediated endocytosis or through transport across the blood brain barrier. ${ }^{67,68} \mathrm{CD} 33$ acts as an endocytic receptor and mediates endocytosis through a clathrin-independent mechanism. ${ }^{50} \mathrm{CD} 2 \mathrm{AP}$ forms a complex with cortactin and regulates internalization and trafficking of a variety of transmembrane proteins. ${ }^{53}$

Genetically directed changes in one or more of the aforementioned genes may result in perturbations of endocytosis and/or synaptic vesicle recycling, and thereby contribute to disease pathogenesis. There may also be important connections between immune and lipid pathways and synaptic dysfunction. For example, activation of the components of the complement system marks unwanted synapses for elimination during development ${ }^{69}$; thus, changes in the complement system by genetic risk variants could accelerate programmed synaptic loss. Dysregulation of cholesterol/lipid metabolism by AD risk genes also could result in synaptic dysfunction because cholesterol promotes synapse formation and is a major constituent of neuronal membranes.

\section{FRONTOTEMPORAL LOBAR DEGENERATION (FTLD, FTD)}

Frontotemporal lobar degeneration is a genetically and pathologically heterogeneous neurodegenerative disorder in which the frontal and temporal lobes preferentially degenerate (for review, see ${ }^{70-72}$ ). Age of onset is typically earlier than in AD. In those with dementia beginning before age 65, FTLD is the second most common cause of primary dementia after AD. Three major phenotypes comprise FTLD: the classic behavioral variant, which is the most common form; progressive nonfluent aphasia; and semantic dementia. Compared with $\mathrm{AD}$, a higher percentage of FTLD is "genetic." Almost 50\% of persons afflicted with FTLD have a positive family history, conforming to an autosomal dominant pattern of inheritance in roughly $10-20 \%$.

In reality, FTLD represents a spectrum of diseases characterized by regional brain degeneration. The spectrum extends from ALS at one end (with or without prominent features of FTLD), through the above-mentioned variants, to corticobasal syndrome and progressive supranuclear palsy at the other end, in which the basal ganglia are involved. Mutations in six unrelated genes have been directly implicated in FTLD, all causing disease in an autosomal dominant manner. Three are approximately equally prevalent, more common than the others: the tau gene $M A P T$, the progranulin gene $G R N$, and the recently described hexanucleotide repeat expansion on chromosome 9, C9ORF72. In various series of FTD patients, the MAPT and $G R N$ genes are each responsible for between $\sim 5$ and $15 \%$ of cases. Because the C9OFR72 repeat expansion was only reported this year ${ }^{100,101}$, its frequency in dominantly inherited and sporadic FTD still remains to be determined, though studies already suggest it is one of the most common causes of FTD. The other three disease genes are much rarer causes of FTLD: TADRP, VCP and $C H M P 2 B$. Clinical genetic testing 
is available for mutations in $M A P T, G R N$, and $V C P$ in the United States and Europe, and for TADRP and CHMP2B in Europe.

Frontotemporal lobe degeneration is proving to be quite complex, both genetically and pathologically. Unlike $\mathrm{AD}$, in which the mutated genes all reside in the same biological pathway and cause essentially identical disease at the neuropathological level, FTLD can be separated into several distinct neuropathological entities reflecting at least two different proteinopathies. One major subset of patients has tau-based protein accumulations (neurofibrillary tangles); this subset represents FTLD caused by tauopathy. ${ }^{73}$ The other major subset has ubiquitin-positive inclusions that are tau-negative yet positive for the recently described disease protein, TDP-43. Because mutations in the gene encoding TDP43 can directly cause FTLD or ALS, this subgroup of FTLD is now considered a "TDP43opathy." "74 Ultimately, this still unfolding story will prove to be even more complicated: there is at least one more FTLD subgroup with ubiquitin-positive inclusions that are negative for both tau and TDP-43, yet positive for FUS, the newest protein on the FTD spectrum. ${ }^{75,76}$

The first chromosomal locus identified in FTLD mapped to chromosome 17, hence the designation FTDP-17 (P for parkinsonism). Soon, mutations in the tau gene, MAPT, were found to cause disease in many persons with FTDP-17. Patients with tau-mediated neurodegeneration always display neurofibrillary tangles. A wide variety of dominantly acting mutations have been discovered in $M A P T$, for which testing is available. Mutations in $M A P T$ cause the protein to abnormally accumulate and form fibrils within neurons. The discovery that $M A P T$ mutations cause dementia firmly established that abnormalities in tau are sufficient to induce neurodegeneration in humans, and indicated that the tau pathology of $\mathrm{AD}$ is as central to $\mathrm{AD}$ pathogenesis as amyloid (for review, $\mathrm{see}^{73}$ ).

Many FTD families whose disease maps to chromosome 17 have neither tau neuropathology nor mutations in $M A P T$. Instead, these families display neuronal inclusions containing both ubiquitin and the TADRP protein, TDP-43. Disease in these families is caused by mutations in the $G R N$ gene encoding progranulin, which resides very near MAPT on chromosome 17. All $G R N$ mutations appear to cause a loss of function of the mutated allele, ranging from nonsense mutations to full gene deletions (for review, see ${ }^{70}$ ). It is now generally accepted that $G R N$ mutations cause disease through haploinsufficiency, but it remains a mystery why partial loss of this protein, linked both to neurotrophic and inflammatory pathways, causes neurodegeneration in which TDP-43 accumulates. In persons with $G R N$ mutations, levels of the granulin protein are significantly lower in peripheral blood cells than in normal controls. ${ }^{77-79}$ This simple "blood test" may eventually serve as a disease test. Genetic testing is available for $G R N$ mutations.

Considerable attention has been paid to the disease protein, TDP43, encoded by the TADRP gene. ${ }^{74,80}$ The TDP43 protein has captured the attention of scientists both because inclusions containing it now define a large subset of neurodegenerative disorders in the FTLD/ALS spectrum, and because mutations in TADRP can cause ALS, ALS/FTLD, or even corticobasal syndrome. Normally a nuclear protein, TDP43 tends to concentrate in the cytoplasm of neurons in affected individuals. ${ }^{81}$ While mutations in TADRP may be the most common cause of familial ALS, they contribute less frequently to FTLD.

A chromosome 9p locus for familial FTD has been known for some time. This year, two groups independently reported an expansion of a non-coding GGGGCC hexanucleotide repeat in a gene known as C9ORF72 as a cause of familial ALS/FTD ${ }^{100,101}$. This repeat expansion becomes the latest in a long line of repeat expansions underlying inherited neurodegenerative diseases. FTD caused by this expansion is a TDP43-opathy. The 
expansion also appears to be a common cause of sporadic and familial ALS. While the hexanucleotide expansion may cause disease via a dominant RNA toxicity manner, much more work is required to determine whether that is the primary pathogenic mechanism.

In a patient with features of FTD and motor weakness, the FTD/ALS spectrum probably will first come to mind as a possible diagnosis. However, if the weakness reflects myopathic changes, one should consider a recently described condition known as "inclusion body myopathy associated with Paget disease of bone and/or frontotemporal dementia" (IBMPFD). As its name suggests, IBMPFD is a remarkably pleiotropic disorder. It is relatively rare and inherited in an autosomal dominant manner. Not all affected individuals with IBMPFD will develop signs of FTD, and particular mutations seem more prone to cause brain degeneration. Profound, progressive language deficits with relatively preserved memory seem to be a common presentation of the dementia in this disease. IBMPFD should be considered in any patient with mid-adult onset dementia accompanied by proximal and distal muscle weakness and/or features suggestive of Paget disease of the bone (including spine or hip pain, pathological fractures or deformities in long bones, increased alkaline phosphatase).

IBMPFD is caused by mutations in Valosin Containing Protein (VCP), an ATPase and ubiquitin binding protein linked to many ubiquitin-dependent cellular pathways, including protein degradation by the proteasome. ${ }^{82}$ Neuropathologically, IBMPFD is a TDP43postive, tau-negative form of FTLD. If there is a family history of similar disease (not necessarily involving all three clinical elements of the disease), then gene testing should be considered and is clinically available. About $80 \%$ of affected persons have a family history consistent with autosomal dominant disease while another $20 \%$ develop sporadic disease. Thus, in the right clinical scenario a positive family history is not essential to consider the diagnosis.

FTD caused by a mutation in the $C H M P 2 B$ (charged multivesicular body protein $2 \mathrm{~B}$ ) gene is very rare - reported in a single Dutch family. Gene testing is not commercially available. The mutation, which is thought to act in a dominant-negative manner, alters a protein component of the endosomal sorting complex known as ESCRT-III, which is required for formation of the multivesicular body, an early precursor to the lysosome ${ }^{83,84}$ Its rarity notwithstanding, the existence of CHMP2B dementia highlights the importance of membrane dynamics and autophagy in neurodegenerative disease. Inclusions in this form of FTLD are negative both for tau and for TDP43.

A newly described, dominant X-linked form of ALS that also can manifest as FTD has been identified in the gene $U B Q L N 2^{102}$. This is a rare cause of familial X-linked ALS which in a minority of cases manifests with an FTD-like dementia. The Ubiquilin 2 protein is functionally linked both to the ubiquitin-proteasome degradation pathway and molecular chaperones. All five described mutations to date occur in a PXX motif near the carboxy terminus of the protein. These mutations cause ubiquilin 2 to accumulate inside motor neurons and hippocampal neurons in skein-like inclusions; Ubiquilin 2 deposits are often associated with TDP43 accumulations. While the pathogenic mechanism in this X-linked form of ALS/dementia is not clear, it could represent a protein aggregation disorder like many other neurodegenerative diseases. Alternatively, mutations may alter ubiquitin 2 function in a manner that perturbs ubiquitin-dependent proteostasis in neurons.

Results of GWAS in FTD began to surface this past year. A newly defined disease susceptibility locus maps to Chromosome 7p21, in the gene TMEM106B. ${ }^{85}$ This linkage was confirmed in a second study, though another failed to identify this locus. While the mechanism by which TMEM106B contribute to one's genetic risk of developing FTLD is 
unknown, certain SNPs in TMEM106B appear to significantly reduce disease penetrance in a cohort of patients with documented $G R N$ mutations, possibly by modulating levels of granulin expression. ${ }^{86}$

\section{DEMENTIA WITH LEWY BODIES (DLB, LBD)}

Synucleinopathies include the overlapping spectrum of Parkinson's disease (PD), PD with dementia, and dementia with Lewy bodies (also known as Lewy body dementia). A key hallmark in these related disorders is the intraneuronal cytoplasmic inclusion known as the Lewy body, in which a-synuclein is the key aggregated protein. In PD, Lewy bodies are largely confined to the substantia nigra and certain regions of the brainstem. In DLB, however, the Lewy bodies are found widespread in the cerebral cortex and are associated with the various cognitive and psychiatric features of Lewy body dementia (progressive cognitive decline, visual hallucinations, fluctuating level of attention and alertness). Thus, one can consider the synucleinopathies as a spectrum extending from pure idiopathic PD through PD with dementia to bona fide Lewy body dementia.

Most DLB is not familial. The most strongly implicated gene in familial DLB is the gene encoding a-synuclein itself (for review, see ${ }^{87}$ ). Triplication of the gene is associated with PD as well as DLB. Rare autosomal dominant mutations in synuclein also can cause familial PD in which dementia is a prominent feature. Glucocerebrosidase mutations implicated in PD also may contribute to DLB. ${ }^{88}$

\section{Prion Dementia}

Most prionopathies are sporadic and non-familial. However, some cases of CreutzfeldtJakob disease (CJD), and other prion disorders, including Gerstmann-Straussler-Scheinker disease (GSS) and fatal familial insomnia, reflect dominantly inherited mutations in the prion gene (for review, see ${ }^{89}$ ). In such families, these are highly penetrant mutations that act dominantly. In sporadic CJD and in variant CD ("mad cow disease"), the sole genetic factor implicated thus far is the prion polymorphism at codon $129 .{ }^{90}$ Codon 129 encodes either valine or methionine. In general among the prion disorders, individuals who are heterozygous for the codon 129 polymorphism have reduced risk for developing sporadic or acquired disease.

Several recent studies ascribe prion-like behavior to other neurodegenerative disease proteins, including tau, $a$-synuclein, and polyglutamine proteins. ${ }^{91-93 ~ " P r i o n-l i k e " ~ i m p l i e s ~}$ the capacity of an abnormally folded polypeptide to recruit another related polypeptide to adopt the same abnormal conformation, initiating a self-amplifying cascade that can even spread from cell to cell and region to region. Whether this is an important element of disease pathogenesis in a wide variety of neurodegenerative proteinopathies is far from certain. But a compelling case can be made that such a phenomenon could underlie the regional spread of degeneration observed in many tauopathies, including FTD.

CADASIL—Cerebral autosomal dominant arteriopathy with subcortical infarcts and leukoencephalopathy (CADASIL) is a dominantly inherited disorder characterized by strokes or transient ischemic attacks (TIAs) in mid-adult life and often preceded by a history of migraine (for review, see ${ }^{94}$ ). It is the most common hereditary cause of stroke and a less common cause of dementia. The brain MRI reveals extensive white matter changes that can be confused with the radiographic pattern of multiple sclerosis. In the evaluation of someone with dementia, a brain MRI revealing extensive white matter disturbances with a history of early strokes or TIAs, and/or history of migraine, should prompt the neurologist to ask in detail about any family history of stroke, multiple sclerosis, dementia, or migraine. In the right clinical setting, genetic testing is appropriate. Mutations in the Notch3 gene cause 
CADASIL. Mutations lead Notch 3 protein to accumulate abnormally in smooth muscle cells of cerebral and extracerebral vessels.

\section{HUNTINGTON'S DISEASE (HD) AND OTHER REPEAT EXPANSION DISORDERS}

The most common of the nine known polyglutamine disorders, Huntington's disease (HD) is a dominantly inherited disorder characterized by the clinical triad of progressive cognitive impairment culminating in dementia, psychiatric disturbance and motor manifestations, most notably chorea (for review see ${ }^{95}$ ). The characteristic motor manifestations, a positive family history for similar disease, and caudate atrophy are among the strong clues that someone may have HD. In late onset HD due to sporadic or new mutations, however, there is no family history. Typically, when HD appears as a sporadic late-onset disorder, the motor manifestations including chorea are prominent enough that the neurologist is clued in to test for HD. The neurologist should also be clued into the possibility that a progressive dementing disorder accompanied by dyskinesias, in which the chronic use of antipsychotics may have led to an assumption that the movements represent tardive dyskinesia, could actually be HD. All patients with HD have a pathogenic CAG repeat expansion which can be assessed through a sensitive and highly specific gene test. Rare phenocopies of HD (e.g., HDL2) should be considered if the HD gene test proves to be negative. ${ }^{96}$

Most other polyglutamine diseases do not manifest with dementia, but rather with ataxia or motor neuron involvement. The one clear exception to this rule is spinocerebellar ataxia type 17 (SCA17). Most individuals with SCA17 have significant cortical involvement and develop progressive cognitive impairment if not frank dementia. ${ }^{97}$ Typically, however, SCA17 includes motor manifestations such as ataxia or dystonia.

The Fragile X premutation tremor ataxia syndrome, or FXTAS, is a disorder typically afflicting males late in life. ${ }^{98,99}$ It most commonly manifests with an essential-type tremor, ataxia, and cognitive impairment. The phenotypic variability is great, however, and some individuals will have a progressive dementia with a gait imbalance or parkinsonism. In an elderly male with cognitive impairment who has a daughter with premature ovarian failure and/or a grandson (through his daughter) with mental retardation, the physician should consider FXTAS in the differential diagnosis. A common, but not pathognomonic, radiologic hallmark is $\mathrm{T} 2$ hyperintensities in the middle cerebellar peduncles. Testing for the FXTAS mutation (a premutation expansion in the Fragile X mental retardation FMR1 gene) is clinically available.

\section{REFERENCES}

1. Bertram L, Lill CM, Tanzi RE. The genetics of Alzheimer disease: back to the future. Neuron. 2010; 68(2):270-281. [PubMed: 20955934]

2. Van Broeckhoven C. The future of genetic research on neurodegeneration. Nat Med. 2010; 16(11): 1215-1217. [PubMed: 21052076]

3. Crews L, Masliah E. Molecular mechanisms of neurodegeneration in Alzheimer's disease. Hum Mol Genet. 2010; 19(R1):R12-R20. [PubMed: 20413653]

4. Kim J, Basak JM, Holtzman DM. The role of apolipoprotein E in Alzheimer's disease. Neuron. 2009; 63(3):287-303. [PubMed: 19679070]

5. Green RC, Roberts JS, Cupples LA, et al. REVEAL Study Group. Disclosure of APOE genotype for risk of Alzheimer's disease. N Engl J Med. 2009; 361(3):245-254. [PubMed: 19605829]

6. Ashe KH, Zahs KR. Probing the biology of Alzheimer's disease in mice. Neuron. 2010; 66(5):631645. [PubMed: 20547123] 
7. Kim D, Tsai LH. Bridging physiology and pathology in AD. Cell. 2009; 137(6):997-1000. [PubMed: 19524503]

8. Thinakaran G, Koo EH. Amyloid precursor protein trafficking, processing, and function. J Biol Chem. 2008; 283(44):29615-29619. [PubMed: 18650430]

9. LaFerla FM. Pathways linking Abeta and tau pathologies. Biochem Soc Trans. 2010; 38(4):993995. [PubMed: 20658991]

10. Ittner LM, Ke YD, Delerue F, et al. Dendritic function of tau mediates amyloid-beta toxicity in Alzheimer's disease mouse models. Cell. 2010; 142(3):387-397. [PubMed: 20655099]

11. Cruchaga C, Kauwe JS, Mayo K, et al. SNPs associated with cerebrospinal fluid phospho-tau levels influence rate of decline in Alzheimer's disease. PLoS Genet. 2010; 6(9):ii, e100110.

12. Fagan AM, Head D, Shah AR, et al. Decreased cerebrospinal fluid Abeta(42) correlates with brain atrophy in cognitively normal elderly. Ann Neurol. 2009; 65(2):176-183. [PubMed: 19260027]

13. Holtzman DM. Cerebrospinal fluid beta-amyloid 42, Tau, and P-tau: confirmation now realization. Arch Neurol. 2009; 66(12):1552-1553. [PubMed: 20008662]

14. Hu WT, Chen-Plotkin A, Arnold SE, et al. Novel CSF biomarkers for Alzheimer's disease and mild cognitive impairment. Acta Neuropathol. 2010; 119(6):669-678. [PubMed: 20232070]

15. Jack CR Jr, Knopman DS, Jagust WJ, et al. Hypothetical model of dynamic biomarkers of the Alzheimer's pathological cascade. Lancet Neurol. 2010; 9(1):119-128. [PubMed: 20083042]

16. Jagust WJ, Landau SM, Shaw LM, et al. Alzheimer's Disease Neuroimaging Initiative. Relationships between biomarkers in aging and dementia. Neurology. 2009; 73(15):1193-1199. [PubMed: 19822868]

17. Morris JC, Roe CM, Grant EA, et al. Pittsburgh compound B imaging and prediction of progression from cognitive normality to symptomatic Alzheimer disease. Arch Neurol. 2009; 66(12):1469-1475. [PubMed: 20008650]

18. Vemuri P, Wiste HJ, Weigand SD, et al. Alzheimer's Disease Neuroimaging Initiative. Serial MRI and CSF biomarkers in normal aging, MCI, and AD. Neurology. 2010; 75(2):143-151. [PubMed: 20625167]

19. Sperling RA, Aisen PS, Beckett LA, et al. Toward defining the preclinical stages of Alzheimer's disease: recommendations from the National Institute on Aging-Alzheimer's Association workgroups on diagnostic guidelines for Alzheimer's disease. Alzheimers Dement. 2011; 7(3): 280-292. [PubMed: 21514248]

20. Bertram L, McQueen MB, Mullin K, Blacker D, Tanzi RE. Systematic meta-analyses of Alzheimer disease genetic association studies: the AlzGene database. Nat Genet. 2007; 39(1):17-23. [PubMed: 17192785]

21. Colhoun HM, McKeigue PM, Davey Smith G. Problems of reporting genetic associations with complex outcomes. Lancet. 2003; 361(9360):865-872. [PubMed: 12642066]

22. Harold D, Abraham R, Hollingworth P, et al. Genome-wide association study identifies variants at CLU and PICALM associated with Alzheimer's disease. Nat Genet. 2009; 41(10):1088-1093. [PubMed: 19734902]

23. Lambert JC, Heath S, Even G, et al. European Alzheimer's Disease Initiative Investigators. Genome-wide association study identifies variants at CLU and CR1 associated with Alzheimer's disease. Nat Genet. 2009; 41(10):1094-1099. [PubMed: 19734903]

24. Seshadri S, Fitzpatrick AL, Ikram MA, et al. CHARGE Consortium; GERAD1 Consortium; EADI1 Consortium. Genome-wide analysis of genetic loci associated with Alzheimer disease. JAMA. 2010; 303(18):1832-1840. [PubMed: 20460622]

25. Hollingworth P, Harold D, Sims R, et al. Alzheimer's Disease Neuroimaging Initiative; CHARGE consortium; EADI1 consortium. Common variants at ABCA7, MS4A6A/MS4A4E, EPHA1, CD33 and CD2AP are associated with Alzheimer's disease. Nat Genet. 2011; 43(5):429-435. 10.1038/ng.803. [PubMed: 21460840]

26. Bettens K, Sleegers K, Van Broeckhoven C. Current status on Alzheimer disease molecular genetics: from past, to present, to future. Hum Mol Genet. 2010; 19(R1):R4-R11. [PubMed: 20388643] 
27. Yerbury JJ, Poon S, Meehan S, et al. The extracellular chaperone clusterin influences amyloid formation and toxicity by interacting with prefibrillar structures. FASEB J. 2007; 21(10):23122322. [PubMed: 17412999]

28. Kirszbaum L, Bozas SE, Walker ID. SP-40,40, a protein involved in the control of the complement pathway, possesses a unique array of disulphide bridges. FEBS Lett. 1992; 297(1-2):70-76. [PubMed: 1551440]

29. Thambisetty M, Simmons A, Velayudhan L, et al. Association of plasma clusterin concentration with severity, pathology, and progression in Alzheimer disease. Arch Gen Psychiatry. 2010; 67(7): 739-748. [PubMed: 20603455]

30. Calero M, Rostagno A, Frangione B, Ghiso J. Clusterin and Alzheimer's disease. Subcell Biochem. 2005; 38:273-298. [PubMed: 15709484]

31. Braskie MN, Jahanshad N, Stein JL, et al. Common Alzheimer's disease risk variant within the CLU gene affects white matter microstructure in young adults. J Neurosci. 2011; 31(18):67646770. [PubMed: 21543606]

32. Krych-Goldberg M, Atkinson JP. Structure-function relationships of complement receptor type 1. Immunol Rev. 2001; 180:112-122. [PubMed: 11414353]

33. Zanjani H, Finch CE, Kemper C, et al. Complement activation in very early Alzheimer disease. Alzheimer Dis Assoc Disord. 2005; 19(2):55-66. [PubMed: 15942322]

34. Eikelenboom P, Veerhuis R, Scheper W, Rozemuller AJ, van Gool WA, Hoozemans JJ. The significance of neuroinflammation in understanding Alzheimer's disease. J Neural Transm. 2006; 113(11):1685-1695. [PubMed: 17036175]

35. Rogers J, Li R, Mastroeni D, et al. Peripheral clearance of amyloid beta peptide by complement C3-dependent adherence to erythrocytes. Neurobiol Aging. 2006; 27(12):1733-1739. [PubMed: 16290270]

36. Brouwers N, Van Cauwenberghe C, Engelborghs S, et al. Alzheimer risk associated with a copy number variation in the complement receptor 1 increasing $\mathrm{C} 3 \mathrm{~b} / \mathrm{C} 4 \mathrm{~b}$ binding sites. Mol Psychiatry. Mar 15.2011 [Epub ahead of print].

37. Tebar F, Bohlander SK, Sorkin A. Clathrin assembly lymphoid myeloid leukemia (CALM) protein: localization in endocytic-coated pits, interactions with clathrin, and the impact of overexpression on clathrin-mediated traffic. Mol Biol Cell. 1999; 10(8):2687-2702. [PubMed: 10436022]

38. Koo EH, Squazzo SL. Evidence that production and release of amyloid beta-protein involves the endocytic pathway. J Biol Chem. 1994; 269(26):17386-17389. [PubMed: 8021238]

39. Carey RM, Balcz BA, Lopez-Coviella I, Slack BE. Inhibition of dynamin-dependent endocytosis increases shedding of the amyloid precursor protein ectodomain and reduces generation of amyloid beta protein. BMC Cell Biol. 2005; 6:30. [PubMed: 16095541]

40. Harel A, Wu F, Mattson MP, Morris CM, Yao PJ. Evidence for CALM in directing VAMP2 trafficking. Traffic. 2008; 9(3):417-429. [PubMed: 18182011]

41. Sakamuro D, Elliott KJ, Wechsler-Reya R, Prendergast GC. BIN1 is a novel MYC-interacting protein with features of a tumour suppressor. Nat Genet. 1996; 14(1):69-77. [PubMed: 8782822]

42. Wigge P, McMahon HT. The amphiphysin family of proteins and their role in endocytosis at the synapse. Trends Neurosci. 1998; 21(8):339-344. [PubMed: 9720601]

43. Meunier B, Quaranta M, Daviet L, Hatzoglou A, Leprince C. The membrane-tubulating potential of amphiphysin 2/BIN1 is dependent on the microtubule-binding cytoplasmic linker protein 170 (CLIP-170). Eur J Cell Biol. 2009; 88(2):91-102. [PubMed: 19004523]

44. Tanaka N, Abe-Dohmae S, Iwamoto N, Yokoyama S. Roles of ATP-binding cassette transporter A7 in cholesterol homeostasis and host defense system. J Atheroscler Thromb. 2011; 18(4):274281. [PubMed: 21173549]

45. Ikeda Y, Abe-Dohmae S, Munehira Y, et al. Posttranscriptional regulation of human ABCA7 and its function for the apoA-I-dependent lipid release. Biochem Biophys Res Commun. 2003; 311(2): 313-318. [PubMed: 14592415]

46. Langmann T, Mauerer R, Zahn A, et al. Real-time reverse transcription-PCR expression profiling of the complete human ATP-binding cassette transporter superfamily in various tissues. Clin Chem. 2003; 49(2):230-238. [PubMed: 12560344] 
47. Jehle AW, Gardai SJ, Li S, et al. ATP-binding cassette transporter A7 enhances phagocytosis of apoptotic cells and associated ERK signaling in macrophages. J Cell Biol. 2006; 174(4):547-556. [PubMed: 16908670]

48. Liang Y, Buckley TR, Tu L, Langdon SD, Tedder TF. Structural organization of the human MS4A gene cluster on Chromosome 11q12. Immunogenetics. 2001; 53(5):357-368. [PubMed: 11486273]

49. Tateno H, Li H, Schur MJ, et al. Distinct endocytic mechanisms of CD22 (Siglec-2) and Siglec-F reflect roles in cell signaling and innate immunity. Mol Cell Biol. 2007; 27(16):5699-5710. [PubMed: 17562860]

50. Crocker PR, Paulson JC, Varki A. Siglecs and their roles in the immune system. Nat Rev Immunol. 2007; 7(4):255-266. [PubMed: 17380156]

51. Dustin ML, Olszowy MW, Holdorf AD, et al. A novel adaptor protein orchestrates receptor patterning and cytoskeletal polarity in T-cell contacts. Cell. 1998; 94(5):667-677. [PubMed: 9741631]

52. Lynch DK, Winata SC, Lyons RJ, et al. A Cortactin-CD2-associated protein (CD2AP) complex provides a novel link between epidermal growth factor receptor endocytosis and the actin cytoskeleton. J Biol Chem. 2003; 278(24):21805-21813. [PubMed: 12672817]

53. Pimplikar SW, Nixon RA, Robakis NK, Shen J, Tsai LH. Amyloid-independent mechanisms in Alzheimer's disease pathogenesis. J Neurosci. 2010; 30(45):14946-14954. [PubMed: 21068297]

54. McGeer EG, McGeer PL. Innate immunity in Alzheimer's disease: a model for local inflammatory reactions. Mol Interv. 2001; 1(1):22-29. [PubMed: 14993335]

55. Bates KA, Verdile G, Li QX, et al. Clearance mechanisms of Alzheimer's amyloid-beta peptide: implications for therapeutic design and diagnostic tests. Mol Psychiatry. 2009; 14(5):469-486. [PubMed: 18794889]

56. Blasko I, Stampfer-Kountchev M, Robatscher P, Veerhuis R, Eikelenboom P, GrubeckLoebenstein B. How chronic inflammation can affect the brain and support the development of Alzheimer's disease in old age: the role of microglia and astrocytes. Aging Cell. 2004; 3(4):169176. [PubMed: 15268750]

57. Rowe JA, Moulds JM, Newbold CI, Miller LHP. P. falciparum rosetting mediated by a parasitevariant erythrocyte membrane protein and complement-receptor 1. Nature. 1997; 388(6639):292295. [PubMed: 9230440]

58. Dietschy JM, Turley SD. Cholesterol metabolism in the brain. Curr Opin Lipidol. 2001; 12(2): 105-112. [PubMed: 11264981]

59. Puglielli L, Tanzi RE, Kovacs DM. Alzheimer's disease: the cholesterol connection. Nat Neurosci. 2003; 6(4):345-351. [PubMed: 12658281]

60. Miwa Y, Takiuchi S, Kamide K, et al. Insertion/deletion polymorphism in clusterin gene influences serum lipid levels and carotid intima-media thickness in hypertensive Japanese females. Biochem Biophys Res Commun. 2005; 331(4):1587-1593. [PubMed: 15883054]

61. Ishikawa Y, Akasaka Y, Ishii T, et al. Distribution and synthesis of apolipoprotein J in the atherosclerotic aorta. Arterioscler Thromb Vasc Biol. 1998; 18(4):665-672. [PubMed: 9555874]

62. Tanaka N, Abe-Dohmae S, Iwamoto N, Yokoyama S. Roles of ATP-binding cassette transporter A7 in cholesterol homeostasis and host defense system. J Atheroscler Thromb. 2011; 18(4):274281. [PubMed: 21173549]

63. Mack JT, Townsend DM, Beljanski V, Tew KD. The ABCA2 transporter: intracellular roles in trafficking and metabolism of LDL-derived cholesterol and sterol-related compounds. Curr Drug Metab. 2007; 8(1):47-57. [PubMed: 17266523]

64. Brody DL, Magnoni S, Schwetye KE, et al. Amyloid-beta dynamics correlate with neurological status in the injured human brain. Science. 2008; 321(5893):1221-1224. [PubMed: 18755980]

65. Shankar GM, Li S, Mehta TH, et al. Amyloid-beta protein dimers isolated directly from Alzheimer's brains impair synaptic plasticity and memory. Nat Med. 2008; 14(8):837-842. [PubMed: 18568035]

66. Selkoe DJ. Alzheimer's disease. Cold Spring Harb Perspect Biol. 2011; 3(7):ii, a004457.

67. Bartl MM, Luckenbach T, Bergner O, Ullrich O, Koch-Brandt C. Multiple receptors mediate apoJdependent clearance of cellular debris into nonprofessional phagocytes. Exp Cell Res. 2001; 271(1):130-141. [PubMed: 11697889] 
68. Bell RD, Sagare AP, Friedman AE, et al. Transport pathways for clearance of human Alzheimer's amyloid beta-peptide and apolipoproteins $\mathrm{E}$ and $\mathrm{J}$ in the mouse central nervous system. J Cereb Blood Flow Metab. 2007; 27(5):909-918. [PubMed: 17077814]

69. Stevens B, Allen NJ, Vazquez LE, et al. The classical complement cascade mediates CNS synapse elimination. Cell. 2007; 131(6):1164-1178. [PubMed: 18083105]

70. Rademakers R, Rovelet-Lecrux A. Recent insights into the molecular genetics of dementia. Trends Neurosci. 2009; 32(8):451-461. [PubMed: 19640594]

71. Rohrer JD, Guerreiro R, Vandrovcova J, et al. The heritability and genetics of frontotemporal lobar degeneration. Neurology. 2009; 73(18):1451-1456. [PubMed: 19884572]

72. van der Zee J, Sleegers K, Van Broeckhoven C. Invited article: the Alzheimer diseasefrontotemporal lobar degeneration spectrum. Neurology. 2008; 71(15):1191-1197. [PubMed: 18838666]

73. Ballatore C, Lee VM, Trojanowski JQ. Tau-mediated neurodegeneration in Alzheimer's disease and related disorders. Nat Rev Neurosci. 2007; 8(9):663-672. [PubMed: 17684513]

74. Chen-Plotkin AS, Lee VM, Trojanowski JQ. TAR DNA-binding protein 43 in neurodegenerative disease. Nat Rev Neurol. 2010; 6(4):211-220. [PubMed: 20234357]

75. Mackenzie IR, Rademakers R, Neumann M. TDP-43 and FUS in amyotrophic lateral sclerosis and frontotemporal dementia. Lancet Neurol. 2010; 9(10):995-1007. [PubMed: 20864052]

76. Urwin H, Josephs KA, Rohrer JD, et al. FReJA Consortium. FUS pathology defines the majority of tau- and TDP-43-negative frontotemporal lobar degeneration. Acta Neuropathol. 2010; 120(1):3341. [PubMed: 20490813]

77. Coppola G, Karydas A, Rademakers R, et al. Gene expression study on peripheral blood identifies progranulin mutations. Ann Neurol. 2008; 64(1):92-96. [PubMed: 18551524]

78. Ghidoni R, Benussi L, Glionna M, Franzoni M, Binetti G. Low plasma progranulin levels predict progranulin mutations in frontotemporal lobar degeneration. Neurology. 2008; 71(16):1235-1239. [PubMed: 18768919]

79. Sleegers K, Brouwers N, Van Damme P, et al. Serum biomarker for progranulin-associated frontotemporal lobar degeneration. Ann Neurol. 2009; 65(5):603-609. [PubMed: 19288468]

80. Geser F, Martinez-Lage M, Robinson J, et al. Clinical and pathological continuum of multisystem TDP-43 proteinopathies. Arch Neurol. 2009; 66(2):180-189. [PubMed: 19204154]

81. Barmada SJ, Skibinski G, Korb E, Rao EJ, Wu JY, Finkbeiner S. Cytoplasmic mislocalization of TDP-43 is toxic to neurons and enhanced by a mutation associated with familial amyotrophic lateral sclerosis. J Neurosci. 2010; 30(2):639-649. [PubMed: 20071528]

82. Weihl CC. Valosin containing protein associated fronto-temporal lobar degeneration: clinical presentation, pathologic features and pathogenesis. Curr Alzheimer Res. 2011; 8(3):252-260. [PubMed: 21222596]

83. Urwin H, Ghazi-Noori S, Collinge J, Isaacs A. The role of CHMP2B in frontotemporal dementia. Biochem Soc Trans. 2009; 37(Pt 1):208-212. [PubMed: 19143633]

84. Lee JA, Liu L, Gao FB. Autophagy defects contribute to neurodegeneration induced by dysfunctional ESCRT-III. Autophagy. 2009; 5(7):1070-1072. [PubMed: 19738424]

85. Van Deerlin VM, Sleiman PM, Martinez-Lage M, et al. Common variants at 7p21 are associated with frontotemporal lobar degeneration with TDP-43 inclusions. Nat Genet. 2010; 42(3):234-239. [PubMed: 20154673]

86. Finch N, Carrasquillo MM, Baker M, et al. TMEM106B regulates progranulin levels and the penetrance of FTLD in GRN mutation carriers. Neurology. 2011; 76(5):467-474. [PubMed: 21178100]

87. Bonifati V. Recent advances in the genetics of dementia with lewy bodies. Curr Neurol Neurosci Rep. 2008; 8(3):187-189. [PubMed: 18541113]

88. Clark LN, Kartsaklis LA, Wolf Gilbert R, et al. Association of glucocerebrosidase mutations with dementia with lewy bodies. Arch Neurol. 2009; 66(5):578-583. [PubMed: 19433657]

89. Brown K, Mastrianni JA. The prion diseases. J Geriatr Psychiatry Neurol. 2010; 23(4):277-298. [PubMed: 20938044] 
90. Mead S, Poulter M, Uphill J, et al. Genetic risk factors for variant Creutzfeldt-Jakob disease: a genome-wide association study. Lancet Neurol. 2009; 8(1):57-66. [PubMed: 19081515]

91. Angot E, Steiner JA, Hansen C, Li JY, Brundin P. Are synucleinopathies prion-like disorders? Lancet Neurol. 2010; 9(11):1128-1138. [PubMed: 20846907]

92. Kim J, Holtzman DM. Medicine. Prion-like behavior of amyloid-beta. Science. 2010; 330(6006): 918-919. [PubMed: 21071652]

93. Frost B, Diamond MI. Prion-like mechanisms in neurodegenerative diseases. Nat Rev Neurosci. 2010; 11(3):155-159. [PubMed: 20029438]

94. Chabriat H, Joutel A, Dichgans M, Tournier-Lasserve E, Bousser MG. Cadasil. Lancet Neurol. 2009; 8(7):643-653. [PubMed: 19539236]

95. Ross CA, Tabrizi SJ. Huntington's disease: from molecular pathogenesis to clinical treatment. Lancet Neurol. 2011; 10(1):83-98. [PubMed: 21163446]

96. Wild EJ, Mudanohwo EE, Sweeney MG, et al. Huntington's disease phenocopies are clinically and genetically heterogeneous. Mov Disord. 2008; 23(5):716-720. [PubMed: 18181206]

97. Stevanin G, Brice A. Spinocerebellar ataxia 17 (SCA17) and Huntington's disease-like 4 (HDL4). Cerebellum. 2008; 7(2):170-178. [PubMed: 18418687]

98. Garcia-Arocena D, Hagerman PJ. Advances in understanding the molecular basis of FXTAS. Hum Mol Genet. 2010; 19(R1):R83-R89. [PubMed: 20430935]

99. Leehey MA. Fragile X-associated tremor/ataxia syndrome: clinical phenotype, diagnosis, and treatment. J Investig Med. 2009; 57(8):830-836.

100. Renton AE, Majounie E, Waite A, et al. A hexanucleotide repeat expansion in C9ORF72 is the cause of chromosome 9p21-linked ALS-FTD. Neuron. 2011; 72(2):257-268. [PubMed: 21944779]

101. DeJesus-Hernandez M, Mackenzie IR, Boeve BF, et al. Expanded GGGGCC hexanucleotide repeat in noncoding region of C9ORF72 causes chromosome 9p-linked FTD and ALS. Neuron. 2011; 72(2):245-256. [PubMed: 21944778]

102. Deng HX, Chen W, Hong ST, et al. Mutations in UBQLN2 cause dominant X-linked juvenile and adult-onset ALS and ALS/dementia. Nature. 2011; 477(7363):211-215. [PubMed: 21857683] 
vascular/traumatic

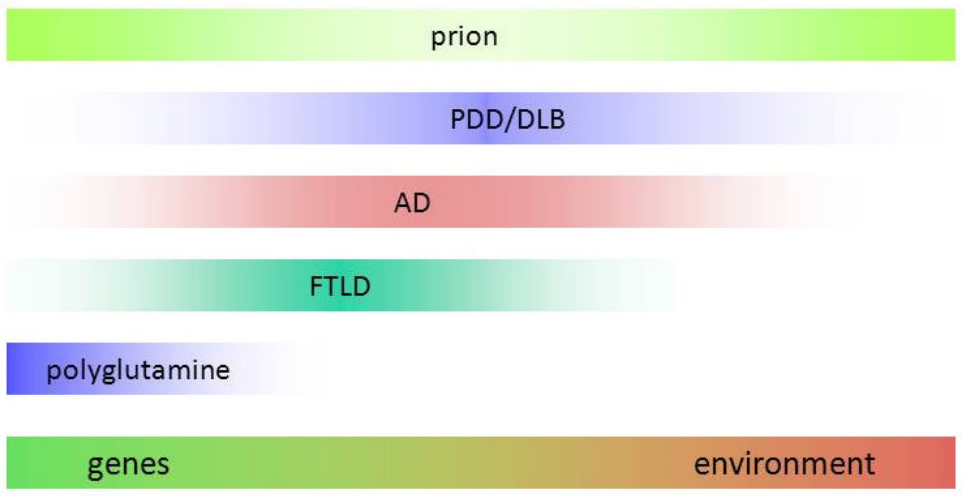

Figure 1.

Spectrum of genes versus environment continuum in the dementias. "Pure" genetic dementias such as Huntington disease (HD) lie at one end of the spectrum, but may be influenced modestly by environmental factors. Conversely, the development of acquired forms of dementia (e.g., chronic traumatic encephalopathy) may ultimately prove to be influenced by genetic factors. Diseases like Alzheimer's disease (AD), frontotemporal dementia (FTD), and dementia with Lewy bodies (DLB) are caused by a mixture of genetic and environmental factors, with rarer familial forms of each of these disorders residing near the genes end of the genes/environment continuum. Prion diseases can be strongly genetic (dominantly inherited familial disease) or acquired (e.g., "mad cow" disease), yet even acquired forms are influenced by a genetic factor in the prion gene. 